Cinémas

Revue d'études cinématographiques

Journal of Film Studies

\title{
Critique/théorie : l'évaluation et la preuve
}

\section{Alain Bergala}

Volume 6, numéro 2-3, printemps 1996

\section{La Critique cinématographique}

URI : https://id.erudit.org/iderudit/1000970ar

DOI : https://doi.org/10.7202/1000970ar

Aller au sommaire du numéro

\section{Éditeur(s)}

Cinémas

\section{ISSN}

1181-6945 (imprimé)

1705-6500 (numérique)

Découvrir la revue

\section{Citer cet article}

Bergala, A. (1996). Critique/théorie : l'évaluation et la preuve. Cinémas, 6(2-3), 29-44. https://doi.org/10.7202/1000970ar

\section{Résumé de l'article}

La spécificité de l'écriture critique, par rapport à celle de la promotion ou de l'analyse, est qu'elle émane de quelqu'un qui s'autorise à " évaluer " et dont une des fonctions est de faite cette évaluation. La morale " scientifique » de l'analyste lui interdit d'évaluer son objet sur le mode critique, conjoncturel. Seul son choix de tel ou tel film laisse supposer - le plus souvent implicitement - une évaluation globale préalable à la décision d'en faite son objet d'étude. L'évaluation - sans laquelle il ne saurait y avoir véritablement d'écriture critique - peut se faite au nom d'un consensus préexistant (autour d'un système de valeurs supposées partagées par les lecteurs de telle revue, tels journal ou hebdomadaire), au nom de la pure subjectivité de son auteur (la critique d'humeur connaît encore de beaux jours) ou en essayant de produire des « preuves " prélevées dans le film pour corroborer la validité des jugements qu'elle émet. Cet article travaille pour l'essentiel ce qui se joue en termes d'évaluation et de preuves, à la frontière (qui a été biographiquement celle de l'auteur) entre la critique et l'analyse. 


\section{Critique/théorie: l'évaluation et la preuve}

\section{Alain Bergala}

\section{RÉSUMÉ}

La spécificité de l'écriture critique, par rapport à celle de la promotion ou de l'analyse, est qu'elle émane de quelqu'un qui s'autorise à "évaluer " et dont une des fonctions est de faire cette évaluation. La morale " scientifique" de l'analyste lui interdit d'évaluer son objet sur le mode critique, conjoncturel. Seul son choix de tel ou tel film laisse supposer - le plus souvent implicitement - une évaluation globale préalable à la décision d'en faire son objet d'étude. Lévaluation sans laquelle il ne saurait $y$ avoir véritablement d'écriture critique - peut se faire au nom d'un consensus préexistant (autour d'un système de valeurs supposées partagées par les lecteurs de telle revue, tels journal ou hebdomadaire), au nom de la pure subjectivité de son auteur (la critique d'humeur connaît encore de beaux jours) ou en essayant de produire des "preuves" prélevées dans le film pour corroborer la validité des jugements qu'elle émet. Cet article travaille pour l'essentiel ce qui se joue en termes d'évaluation et de preuves, à la frontière (qui a été biographiquement celle de l'auteur) entre la critique et l'analyse.

\footnotetext{
ABSTRACT

What makes criticism distinct from promotional or analytic writing is that in critical writing, the author is someone who assumes the authority to "evaluate," whose functions include doing so. The "scientific" morality of the analyst forbids the evaluation of the object of analysis in a critical, conjunctural fashion. Only the choice of which film to analyse signals, most
} 
often implicitly, an overall evaluation prior to the decision to study a particular film. Evaluation, without which one cannot really speak of criticism, may be done in the name of a pre-existing consensus (around a system of values presumably shared by the readers of such and such magazine, newspaper or weekly), or in the name of the pure subjectivity of the author (mood criticism is still in full flower), or by extracting "proofs" from the film to support the validity of the judgments made. This article looks in particular at what is involved in evaluation and proof, at the intersection (reflecting the biography of its author) between criticism and analysis.

Je postulerai ceci: tout texte qui ne se donne pas comme fonction d'évaluer le film ou l'œuvre cinématographique dont il parle n'est pas un texte critique. C'est le seul critère possible pour tracer une ligne de démarcation entre le discours critique et les autres types de discours sur le cinéma: le discours analytiquethéorique (qu'il soit universitaire ou non), le discours promotionnel, le discours informatif. Il importe plus que jamais de tracer nettement cette ligne de démarcation, aujourd'hui où de plus en plus d'écrivants de cinéma supposés occuper un espace critique ont démissionné progressivement - parallèlement à la montée en puissance des discours d'intimidation et de promotion à peine déguisée dans les médias à grande surface — de cette fonction d'évaluation sans laquelle il ne saurait y avoir de véritable dimension critique.

La frontière que je voudrais commencer à explorer ici est plus précisément celle qui sépare le discours critique et le discours d'analyse théorique. Je la connais pour l'avoir personnellement fréquentée sur ses deux rives, d'un côté comme critique aux Cabiers du cinéma, et de l'autre comme enseignant-chercheur de cinéma à l'université. Celui qui vient de la critique et traverse la frontière qui donne sur l'écriture théorique doit d'abord remettre en cause le rapport déontologique qu'il a entretenu jusque-là avec l'obligation d'évaluation. La pression dont il se libère ḍ'un côté l'assignation à juger - , il la retrouve sous une autre forme, celle de l'obligation d'étayer ses hypothèses en convoquant des exemples et en fournissant des preuves de tout ce qu'il avance. 
Si tout critique de cinéma digne de cette fonction se doit d'évaluer les objets dont il parle, les valeurs au nom desquelles il le fait peuvent être principalement de deux natures. Relever de sa propre subjectivité, du droit du critique à juger du film comme spectateur-cobaye ou étalon, qui énonce pour les autres spectateurs, ses lecteurs, les effets (de sens, d'émotion), les réactions (esthétiques, morales, etc.) que la vision de ce film a provoqués en lui. Ou relever d'un consensus préalable, d'un certain nombre de valeurs constituées en système - même implicite auxquelles son jugement critique va s'adosser. Même si tendancieusement, ces deux ordres de valeurs définissent - dans leurs formes radicales - deux types de critiques diamétralement opposées, tout critique de cinéma sait bien qu'il lui faut trouver son propre compromis entre ces deux postulations contradictoires: entre la nécessaire et inévitable subjectivité de ses réactions devant le film et la référence à une configuration de valeurs qui peuvent faire communauté.

\section{La critique à fondement communautaire}

Le consensus préalable - qui implique un système de valeurs collectives fondant l'évaluation critique - peut être de nature sensiblement différente selon l'époque et le support. J'en vois trois catégories essentielles.

1. Un consensus rédactionnel très fort sur les valeurs qui ne sont pas (encore) celles du public. La communauté qui partage ces valeurs est alors réduite (parfois à l'équipe de rédaction ellemême), mais la force d'affirmation et de conviction peut être d'autant plus forte qu'il s'agit de valeurs à imposer, encore minoritaires, voire inexprimées. L'organe de presse devient alors machine de guerre, et ses rédacteurs, les fers de lance de ces nouvelles valeurs. Ce fut historiquement le cas des jeunes Cabiers $d u$ cinéma, non pas à leur création mais au milieu des années 50 . La cohésion de la communauté rédactionnelle est alors renforcée par le sentiment que ses valeurs n'appartiennent pour le moment qu'à elle, ou à une petite minorité. L'évaluation est forte et tranchée puisque le groupe doit tracer la ligne de démarcation la plus nette possible entre les valeurs dominantes et les valeurs nouvelles (les siennes) qu'il entend faire entendre et 


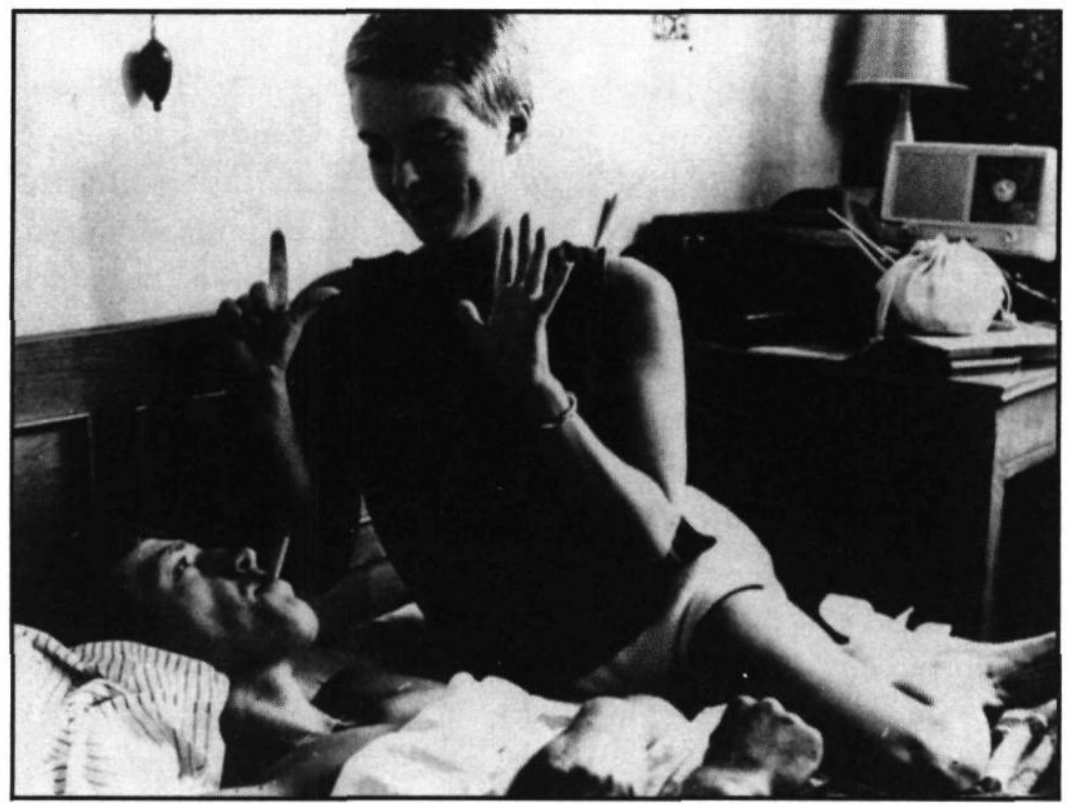

\section{À bout de souffle de Jean-Luc Godard (1959)}

Collection Cinémathèque québécoise

triompher. Cette affirmation forte des valeurs d'un petit groupe, ses évaluations parfois provocantes sont proches de l'ordre toujours un peu terroriste, manichéen et dogmatique - du manifeste. Le texte de François Truffaut, "Une certaine tendance du cinéma français", a été exemplaire de ce type d'intervention critique, tempéré il est vrai par une forte volonté de convaincre avec exemples concrets, preuves à l'appui ${ }^{1}$. Il va de soi que la capacité de pénétration de valeurs nouvelles ne dépend pas seulement de la force de conviction et de la cohésion du petit groupe qui les porte. Pour que ces valeurs trouvent, dans le public, une résonance de masse, il faut qu'une sensibilité diffuse soit prête à les accueillir. Qu'il y ait déjà, même inexprimée jusque-là, une "faille dans le consensus" pour reprendre une expression de Serge Daney (1984). Ce fut le cas pour l'équipe rédactionnelle des Cahiers du cinéma des années 50, dont le mérite a été de formuler en termes d'évaluation cinématographique des valeurs latentes que le public qui avait l'âge de ces jeunes critiques ressentait sans doute confusément: que la 
société française sortait de l'après-guerre (et des valeurs centrées sur le passé) et qu'elle entrait dans une phase nouvelle de consommation, de changement de mœurs, d'émergence de valeurs jeunes, de goûts différents.

2. Un consensus préalable nettement revendiqué entre la revue (l'hebdomadaire, le journal) et ses lecteurs. C'est le cas chaque fois que le lectorat de la revue participe d'un consensus fort sur des valeurs (idéologiques, esthétiques, philosophiques, religieuses, etc.) qui font de l'acte d'achat, d'abonnement et de lecture un geste d'appartenance. C'est le cas aujourd'hui, en France, d'une revue comme Etudes, par exemple, d'origine et d'obédience jésuites ${ }^{2}$, qui intervient de façon régulière et très pointue dans le champ du cinéma. Le critique est alors celui qui va servir de guide "spécialisé». Devant le flux permanent des films, il est en quelque sorte "délégué" en tant que "spécialiste" du spécifique cinématographique - par la communauté idéologique qui englobe les lecteurs et les producteurs de la revue - à transformer en choix cinématographiques les valeurs du groupe. Ce fut longtemps en France le cas de la critique cinématographique dans Télérama, sur un consensus de valeurs que l'on pourrait définir à l'emporte-pièce comme "catholiques libérales cultivées». Plus le consensus préalable entre la revue et ses lecteurs est fort, plus le critique est exposé à de violentes réactions chaque fois que ceux-ci ont l'impression qu'il trahit en traduisant ses valeurs communautaires en choix de cinéma trop personnels, trop déviants, voire contradictoires avec les fondements consensuels du groupe. Le risque est d'autant plus grand que le film touche de près aux valeurs sensibles du groupe. La marge d'évaluation personnelle du critique est alors relativement faible: il est sous surveillance du groupe, mais il a par contre l'avantage sur ses collègues - devant la difficile et angoissante obligation d'évaluer sans tarder et sans recul tout nouveau film - de disposer de critères forts, définis, d'un système de valeurs auquel il peut adosser son jugement, même si cela ne le dispense nullement de s'engager à un certain moment, comme tout critique digne de ce nom, en son âme et conscience.

3. Un consensus plus mou, de nature plus "sociologique" qu'idéologique. Je fais référence ici à une presse "en miroir" 
dont le lectorat, aux limites beaucoup plus floues, participe plus d'une appartenance sociologique globale que d'une communauté véritable autour de valeurs définies: les femmes modernes citadines et idéologiquement "évoluées", par exemple, pour un magazine féminin actuel comme Cosmopolitan. La rubrique "cinéma" $\mathrm{y}$ relève plus d'une brève signalétique que de la critique. Le "critique" de cinéma (qui n'est souvent pas un spécialiste, mais un journaliste du secteur "culture" ou "sorties") n'est plus alors "délégué" par la communauté de ses lecteurs sur le front du cinéma, il est le "miroir " inerte de la catégorie sociologique supposée être celle de ses lecteurs, et qui est en tout cas la "cible" commerciale de la publication. Il est "commis" à repérer les films qui donneront à ses lecteurs une image plaisante, gratifiante, de la fraction de population à laquelle ils appartiennent imaginairement et dont la publication est le miroir, et non le fer de lance. C'est un préposé au consensus. Son slogan pourrait être: nous défendons (ou par défaut d'évaluation: nous signalons) ce que vous aimerez. Sa marge de manœuvre est à peu près nulle dans la mesure où c'est le goût moyen supposé de ses lecteurs qui est son seul critère. S'il est capable d'évaluation forte, et même d'arrogance (tel film d'un grand cinéaste est trop long, il aurait dû le couper, car ses lecteurs à lui vont s'ennuyer), ce n'est jamais en son nom propre mais au nom des valeurs et des droits de ses lecteurs. En France, aujourd'hui, une revue pourtant spécialisée comme Première relève très largement de cette catégorie pour un public jeune, branché, qui se veut seulement informé sur le cinéma qui est censé le concerner, lui plaire sans efforts ni ennui.

Les frontières entre ces trois catégories de critiques sont évidemment très poreuses. Tout critique de cinéma écrit toujours quelque peu "en miroir" par rapport à l'imaginaire qu'il s'est construit de son lectorat. Ne serait-ce que dans le style, le niveau de langage, le vocabulaire. Même si sa pensée du cinéma restait rigoureusement cohérente avec elle-même, et d'une grande homogénéité, André Bazin n'écrivait pas tout à fait de la même plume pour ses lecteurs du Parisien libéré et pour ceux des Cahiers du cinéma. Pas plus que Serge Daney pour ses lecteurs des Cabiers et de Libération. J'ajouterais qu'il n'est pas rare de 
voir une publication - souvent sous l'effet d'un succès public massif - passer de la deuxième catégorie (à consensus préalable fort entre lecteurs et rédacteurs) à la troisième (et devenir le "miroir" plus inerte d'une catégorie sociologique aux valeurs plus floues). Ce fut le cas en France lorsque Télérama est devenu l'hebdomadaire culturel de prédilection d'une petite bourgeoisie cultivée très élargie par rapport à son lectorat antérieur, beaucoup moins définie dans ses convictions religieuses et morales, débordant très largement le noyau des lecteurs qui avaient fondé le consensus catholique initial de cette publication. Il peut arriver aussi que le triomphe des valeurs de combat d'une revue de type "machine de guerre" modifie le rapport initial, militant, à ses lecteurs, en pacte consensuel de la deuxième catégorie. C'est à l'évidence ce qui est arrivé aux Cahiers du cinéma après le triomphe de la Politique des auteurs: ses lecteurs, résolument convaincus du bien-fondé de cette politique, ont attendu de "leur" revue qu'elle confirme les valeurs nouvelles qu'ils avaient assimilées et adoptées.

\section{La critique à fondement subjectif}

Dans le deuxième cas, l'organe de presse ouvre dans ses colonnes un espace nettement localisé de forte liberté rédactionnelle (sur le mode traditionnel de la chronique, du billet d'humeur), une sorte de "concession" donnée à un individu qui a généralement "une signature", un style personnel qui le distinguent du ton moyen du journal ou de la revue. Il s'autorisera à évaluer les films de son seul point de vue subjectif, sans avoir de comptes à rendre à un quelconque consensus préalable. Il n'a même pas à justifier d'un certain nombre de valeurs stables qui pourraient finir par fonder ses évaluations au jour le jour (ou au mois le mois) sur un quelconque "système" personnel. Les lecteurs n'entrent pas avec lui dans un rapport d'identification communautaire mais dans un rapport plus ludique de curiosité et de surprise. Le lecteur le consulte moins comme un guide ("Dois-je aller voir ce film?») que comme un partenaire de conversation imaginaire ("Qu'est-ce qu'il a bien pu penser de ce film? Va-t-il le brûler ou l'encenser?»). Jean-Louis Bory a pratiqué avec beaucoup de conviction et de talent cette critique 
subjective dans Le Nouvel Observateur des années 60. Dans le journal Libération, Gérard Lefort a repris il y a quelques années cette tradition mondaine française du "critique d'humeur" brillant.

Ces critiques ne s'autorisent que d'eux-mêmes pour évaluer les films un par un, tel que l'actualité les livre à une consommation désordonnée. Le critique d'humeur est volontiers excessif (dans la louange comme dans l'opprobre), réactif et paradoxal (il prend plaisir à se singulariser, à ne pas être dans le rang, à choquer). Il aime surprendre, même par rapport aux attentes qu'il a lui-même fini inévitablement par susciter. Il aime briller, il est parfois prêt à sacrifier un film parce qu'il n'a pas su résister à un jeu de mots sur le titre ou à un trait d'esprit qui flatte son narcissisme d'écrivant. Il n'a pas la tête systématique et affiche volontiers sa spontanéité, son imprévisibilité comme antidotes à tous les consensus et à tous les dogmatismes. Il n'hésite pas à porter un jugement tranché, même s'il sait bien qu'il risque d'entrer en contradiction avec les valeurs supposées de son lectorat. Il se situe entre la libre pensée ("Foin de tous les systèmes!») et le narcissisme ("Ce que je pense est forcément intéressant!»).

\section{Le chercheur et son choix d'objet}

Le chercheur, contrairement au critique, n'a aucune obligation d'évaluation explicite de son objet d'études. Ce dont il devra rendre compte, devant ses pairs, c'est d'une cohérence interne: l'adéquation de son objet à sa problématique et aux outils théoriques qu'il a choisi d'utiliser dans ses recherches et ses analyses. Il y a toujours au moins une espérance de "productivité" théorique dans le choix que fait un chercheur de tel ou tel objet d'étude par rapport à son questionnement. Mais contrairement à celui du critique, le choix d'objet du chercheur n'a pas à être fondé par une évaluation "externe", liée à ses goûts propres ou à la conjoncture cinématographique ni même à l'importance historique (artistique ou culturelle) du film ou de l'auteur choisi. A priori, le chercheur semble dégagé de toute impureté conjoncturelle.

Le chercheur doit justifier de la justesse et de l'importance de ses résultats scientifiques, mais en aucun cas des choix d'objets 
qui ont été à l'origine de cette recherche. Au regard de la recherche pure, tout objet d'étude est légitime. Mais dans un domaine où le goût et les affects sont toujours en jeu, ce choix d'objet fait inévitablement signe, sinon sens. Roland Barthes a posé clairement la question:

J'aime, je n'aime pas: cela n'a aucune importance pour personne; cela, apparemment, n'a pas de sens. Et pourtant tout cela veut dire: mon corps n'est pas le même que le vôtre. Ainsi, dans cette écume anarchique des goûts et des dégoûts, sorte de hachurage distrait, se dessine peu à peu la figure d'une énigme corporelle, appelant complicité ou irritation. Ici commence l'intimidation du corps, qui oblige l'autre à me supporter libéralement, à rester silencieux et courtois devant des jouissances ou des refus qu'il ne partage pas (p. 121).

De ce goût personnel, de cette singularité corporelle dont relève toujours, en dernière instance, en deçà de toutes les justifications théoriques, son choix d'objet (j'aime suffisamment tel film ou tel cinéaste pour passer des mois ou des années à travailler sur lui), le chercheur, l'universitaire est exempté de se justifier. De cela même qui fonde psychiquement son rapport à son objet d'études (et dont la vigueur de son investissement dépendra), il ne lui faut pas trop parler, sous peine que les résultats de ses travaux soient soupçonnés d'impureté scientifique, de trouble motivation subjective. S'il en parle - et c'est presque devenu une convention depuis Roland Barthes, justement c'est sur le mode du "lever de rideau", en prélude, en off de la partie sérieuse de son travail: un bref récit autobiographique, une anecdote raconteront avec pudeur une origine volontiers mythique de ce choix d'objet.

Quant à la communauté qui fonde les valeurs qui sont les siennes en tant que chercheur (l'exigence méthodologique, la productivité théorique de son travail, etc.), elle ne s'autorise en apparence aucun jugement de valeur sur l'évaluation implicite d'une œuvre, d'un cinéaste, d'un courant que représente pour un individu l'engagement à long terme pour en faire un objet d'étude. Tout chercheur est réputé libre a priori de son choix d'objet. Pourtant, là aussi, ce choix fera signe: il sera repéré 
comme appartenant à telle ou telle mouvance, tel ou tel "groupe du même goût", héritier de telle ou telle tradition, suscitant par ce simple choix initial les complicités et les irritations dont parle Barthes. Il aura ensuite l'impression de surprendre s'il change radicalement de "catégorie". Il éprouvera parfois la nécessité de s'en expliquer.

\section{La convocation des exemples probants}

Lévaluation critique, sauf lorsqu'elle se veut délibérément subjective ou arrogante, s'efforce toujours - plus ou moins - de produire quelques preuves autovalidantes, prélevées dans le film lui-même. Un jugement de valeur sur un film n'a de sens que s'il fournit au lecteur un minimum de garanties sur le fait que ce film-là relève bien, comme objet, des arguments invoqués pour le critiquer. Le plus souvent, le critique va convoquer en cours de texte quelques exemples (telle scène, tel plan, telle réplique, tel cadrage, tel personnage) prélevés sur le film comme objet descriptible, pour attester que celui-ci se prête objectivement à sa critique, même revendiquée comme partiellement subjective. Ces points de jonction, par lesquels il légitime la rencontre erratique de son discours (fondée sur des valeurs externes) et de l'objetfilm, fonctionnent comme autant de preuves du bien-fondé de son évaluation. Chacun d'eux renoue, de loin en loin, le contrat de confiance tacite entre le critique et son lecteur: "Je pourrais te prouver ce que j'affirme, même si l'espace de ma critique ne me permet de le faire que rapidement et allusivement. "Le théoricien, pour sa part, n'aura jamais droit à ce mode conditionnel ni à la connivence plus ou moins ludique qu'il engage.

Dans ma typologie sommaire des discours critiques, seul le critique d'humeur peut s'arroger le droit a priori de ne convoquer aucun exemple, ni aucune preuve prélevée dans le film luimême, de ce qu'il avance, puisqu'il s'autorise de sa seule subjectivité critique. Dans une affirmation de type "ce film m'a plu/ ce film m'a déplu ", le fondement de la critique est moins le film lui-même que le sujet écrivant comme surface de réaction sensible, qui cherche à entraîner par contagion une adhésion à sa propre réaction, à ses affects face au film, plutôt qu'à les fonder par des preuves supposées objectives. Il n'en reste pas moins que 
même le critique d'humeur éprouve à l'occasion, lui aussi, le besoin de convoquer quelques exemples de ce qu'il aime ou pas dans le film. Ne serait-ce que pour faire exister un tant soit peu dans l'imaginaire de son lecteur (qui souvent ne l'a pas encore vue) l'œuvre dont il parle.

Lorsqu'un critique convoque des preuves sous forme d'exemples, il peut le faire tendancieusement de deux façons bien différentes selon sa visée première. Sur un mode rhétorique s'il s'agit avant tout pour lui de convaincre; sur un mode analytique s'il s'agit avant tout pour lui de prouver. C'est le propre du discours critique, en tant qu'il est nécessairement bref, conjoncturel, sans recul historique ni analytique (souvent le critique n'a vu qu'une fois ou deux le film dont il parle) d'être un discours de compromis entre prouver et convaincre. Les plus pédagogues (pour qui les écrits critiques d'André Bazin restent encore aujourd'hui un modèle inégalé) essaieront toujours de prouver pour convaincre. Les plus "intuitifs-rapides" (Serge Daney a été récemment le plus brillant représentant de cette catégorie) essaieront d'abord de convaincre tout en convoquant secondairement quelques preuves "au passage", à la volée.

La convocation des preuves n'est pas tout à fait du même ordre dans ces deux cas de figure: ceux qui veulent gagner la confiance de leur lecteur avant de l'impliquer dans leur jugement de valeur, ceux qui préferent capter d'abord sa croyance et se justifier ensuite en cours de route. La convocation des preuves est la pièce maîtresse du discours des premiers, qu'elle ralentit: convoquer des exemples (décrire un plan, une scène, un geste d'acteur, raconter un moment du film), les déplier nécessitent toujours d'arrêter pour un moment la dynamique de la pensée. Cette pause ressemble quelque peu à celle qu'implique toute description dans la dynamique d'un récit. Dans le discours des seconds, qui préferent l'emportement de la conviction par la vitesse, la convocation des preuves est de l'ordre du ravitaillement en plein vol. Les premiers prennent le temps d'installer une configuration d'exemples qui constitueront les fondations de leur jugement de valeur. Les seconds pratiquent plus volontiers la tactique du prélèvement exemplaire: un plan suffira parfois à fonder tout un jugement de valeur. On se souvient de 
l'exemple du travelling de Kapo (Pontecorvo, 1961) pour Jacques Rivette ${ }^{3}$ et de celui de la chaussure de L'Amant (JeanJacques Annaud, 1991) pour Serge Daney (1992).

Lorsqu'il convoque un exemple dans le film même, le critique est dans une posture qui le rapproche quelque peu de celle de l'analyste: il ressent l'obligation de valider son discours, sa thèse, du côté de la réalité textuelle du film ou de l'œuvre. La différence fondamentale reste que la convocation de cette preuve par le critique a pour finalité première, ontologiquement impure, de parvenir à convaincre le lecteur de sa bonne foi et du bien-fondé de son évaluation. Le chercheur, lui, est statutairement dispensé d'évaluation, mais il est dans l'obligation scientifique - devant la communauté des autres chercheurs - de fournir des preuves, et des preuves vérifiables, de la validité de ses analyses. Le ravitaillement en plein vol, la convocation d'un seul prélèvement exemplaire lui sont interdits. Il n'a pas droit à l'exercice du « raid théorique" qui a fait les beaux jours des Cabiers du cinéma dans les années 60 et $70^{4}$. Il doit fournir des preuves valables et suffisantes pour asseoir toute affirmation personnelle: la reconnaissance de la valeur scientifique de ses recherches en dépend. L'espace d'écriture et la patience de ses lecteurs lui sont moins chichement comptés: il peut prendre le temps, et le nombre de pages nécessaires, pour convoquer tous les exemples qu'il jugera utiles. Le critique ne répond que devant ses lecteurs (qui n'iront pas vérifier, texte en main, la validité des exemples) qu'il doit convaincre de l'étayage objectif de son jugement de valeur. $\mathrm{Ce}$ qui est en jeu, pour le critique, est en définitive d'emporter la croyance de celui qui le lit, et sa possible adhésion. Le chercheur, pour sa part, n'a pas à convaincre d'abord un lecteur individuel; il doit avant tout rendre des comptes devant la communauté de ses pairs. Ce qui est en jeu, pour lui, c'est aussi d'emporter une croyance, mais il s'agit de celle des autres chercheurs, et elle ne lui sera accordée - peut-être - que s'il fournit des preuves vérifiables, multiples, concordantes et suffisamment développées pour que la cohérence interne de ses thèses soit indiscutable par rapport à son objet d'étude. Tous les deux - le théoricien comme le critique - rencontrent la même butée langagière qui fait que la "citation" littérale est 
impossible dans ce domaine: toute convocation linéaire du texte cinématographique par le langage écrit est forcément traduction, transposition, donc inévitablement trahison. Le problème est connu et repéré en théorie depuis longtemps.

Dans toute convocation d'exemple à fin de preuve, il entre toujours une part - même inconsciente - de tricherie ludique, de fausse innocence. Quelque chose y relève toujours, peu ou prou, du tour de passe-passe: "Regarde ce qu'exhibe avec ostentation ma main droite pendant que ma main gauche tire un voile sur ce que tu dois provisoirement oublier." Le théoricien connaît aussi bien que le critique toutes les ruses et les complaisances intellectuelles qu'autorise la convocation d'exemples probants. Il les pratique à l'occasion (parfois en toute bonne foi par rapport à sa propre morale et à sa vigilance de chercheur), mais à la différence du critique, il sait qu’il écrit sous surveillance et que l'instance symbolique qui le jugera est beaucoup plus vigilante que celle des lecteurs du critique. Le théoricien aussi, finalement, doit emporter une conviction, mais il doit affronter, pour y réussir, des censures plus rigides et une méticulosité beaucoup plus suspicieuse que le critique. La règle du jeu est presque la même mais pas le public qui assiste au tour de passe-passe. Le critique est un prestidigitateur qui fait son tour devant un public d'amateurs plus ou moins éclairés, le théoricien, devant des professionnels qui sont aussi des concurrents. Ce ne sont pas tout à fait les mêmes exhibitions qui satisferont les uns et les autres.

Quant à l'évaluation "externe» du résultat de ses travaux par rapport à l'état de la recherche à un moment donné de l'histoire de la théorie dans sa discipline, elle constituera une deuxième épreuve, mais seulement $s$ 'il a satisfait aux règles de l'homologation précédente. Son travail doit d'abord être jugé recevable par la communauté scientifique avant d'être évalué par comparaison avec celui des autres chercheurs de sa catégorie. Le critique, aurait-il la tête indiscutablement théoricienne (qui le contesterait aujourd'hui pour Bazin, Bonitzer ou Daney?), n'a pas à franchir toutes ces étapes de validation intermédiaires. Entre la temporalité au jour le jour (ou au mois le mois) qui est celle de ses publications éphémères et le jugement à très long terme de 
l'histoire des idées qui décidera seule de la postérité des thèses qu'il a pu avancer - livraison après livraison, sans avoir eu forcément la possibilité d'en dégager un jour le système dans un texte plus synthétique ${ }^{5}$-, il n'a à affronter aucune instance de légitimation. Cette absence structurelle d'homologation n'a pas empêché la pensée bazinienne, éparpillée dans une poussière de textes critiques de circonstances, de se cristalliser en théorie implicite, en un système fort, l'un des plus productifs sans aucun doute qui ait jamais été élaboré dans le champ de la pensée du cinéma. Il est trop tôt pour savoir s'il en ira de même de la pensée de Serge Daney, mais l'on peut déjà constater l'extraordinaire fréquence de convocation de ses idées et de ses concepts dans les travaux de recherche sur le cinéma de ces 10 dernières années.

\section{Pureté et compromission imaginaires}

La visée première d'une écriture critique est immédiatement entachée d'impureté, si on la compare à celle de la recherche. Elle a toujours affaire à un extérieur éphémère qui est l'actualité cinématographique, la conjoncture. Elle a toujours, peu ou prou, la visée d'une efficacité immédiate dans le réel: convaincre, agir sur les choix du public, infléchir ses goûts, emporter la conviction du lecteur au moment même où paraît l'article. L'obligation d'évaluation "à chaud " la met en risque permanent de se tromper devant les choix que fera le temps. Le critique a les mains impures, mais il peut parfois (même si c'est de moins en moins) se flatter d'avoir des mains et d'agir sur le devenir proche de son objet, le cinéma. Ce fut indiscutablement le cas de Bazin avec l'émergence de la Nouvelle Vague. Travaillant sans autre contrôle que celui de sa conscience et de sa réputation professionnelles, il est guetté par toutes les dérives subjectives, les leurres des engouements et des modes passagères, le manque de recul par rapport à son objet.

Le chercheur, l'analyste, peut revendiquer plus facilement, pour sa part d'écriture sur le cinéma, une moindre compromission avec l'actualité, la conjoncture. Il travaille sous contrôle de la communauté de ses pairs et court moins le risque de se fourvoyer dans les chemins dangereux de la subjectivité. Pourtant, 
son intervention agit aussi comme une évaluation, mais dans une autre conjoncture et une autre temporalité, qui sont celles de la mémoire du cinéma.

Écrits critiques et écrits analytiques sur le cinéma participent au bout du compte de l'écriture de l'histoire du cinéma dans la mesure où ils parviennent à jouer (le critique à chaud, le théoricien à froid) un rôle dans l'inscription d'un film, d'un cinéaste, d'un mouvement, dans la mémoire en permanente construction du cinéma. C'est là où le fameux choix d'objet, dont le chercheur est exempté de justification et d'évaluation, participe aussi - qu'il le veuille ou non, qu'il en soit plus ou moins conscient - d'une conjoncture. Un chercheur qui déciderait de publier, en 1996, un livre d'importance sur René Clair ou Dziga Vertov, participerait par ce seul choix d'une réactivation de la présence du cinéaste élu dans la mémoire du cinéma qui est actuellement la nôtre. Il existe aussi une actualité intellectuelle de la recherche, qui n'appartient pas à la même temporalité que l'actualité cinématographique (celle du grand public et des critiques). Elle est le fait de la communauté des chercheurs, historiens, de tous les écrivants de cinéma pour qui la mémoire du cinéma est un terrain d'exercice et un enjeu. Elle a ses valeurs et ses modes, souvent non dites, qui précipitent tel cinéaste, tel film, dans la disparition provisoire, dans un trou noir de la mémoire du cinéma, ou au contraire opèrent telle résurrection imprévisible quelques années seulement auparavant. De cette responsabilité conjoncturelle, le théoricien n'est pas comptable devant ses pairs, même s'il l'est — qu'il le veuille ou non devant le cinéma comme patrimoine culturel.

La recherche, au cinéma, n'est jamais pure. Tout choix d'objet est déjà une évaluation, une façon d'intervenir dans l'ordre des valeurs de jugement. Toute convocation d'exemples probants est à la fois trahison ontologique du texte filmique et manipulation à fin de conviction. Quiconque est passé un jour de la posture de critique à celle de théoricien — dans un sens ou dans l'autre - sait bien par expérience intime que la ligne de démarcation entre la pensée critique et la pensée théorique n'est pas si étanche. Nul doute que la pensée théorique sur le cinéma n’ait besoin — ne serait-ce qu'à cause de l'impureté ontologique de 
son objet — d'un "autre" radicalement compromis dans le conjoncturel, le subjectif et l'obligation d'évaluation, pour se constituer imaginairement comme scientifique. Cette ligne de démarcation, qui est censée se fonder du côté de l'évaluation et de la preuve, s'estompe à vue d'œil au fur et à mesure que l'on s'en approche en arpenteur. C'était le modeste objectif de ce texte.

\section{Université de Rennes}

\section{NOTES}

1 Il reste toujours chez le polémiste François Truffaut quelque chose de l'autodidacte qui aime prouver, convaincre par des exemples, et du disciple d'André Bazin qui aime expliquer. Voir "Le point critique", table ronde, Cahiers du cinéma, n 356 (février 1984).

2 La revue Études a été fondée en 1856 par des pères de la Compagnie de Jésus, 39 ans avant la naissance du cinéma!

3 Dans cette critique du film de Pontecorvo, intitulée "De l'abjection ", Jacques Rivette écrivait : "Voyez cependant, dans Kapo, le plan où Riva se suicide, en se jetant sur les barbelés électrifiés; l'homme qui décide, à ce moment, de faire un travellingavant pour recadrer le cadavre en contre-plongée, en prenant soin d'inscrire exactement la main levée dans un angle de son cadrage final, cet homme n'a droit qu'au plus profond mépris" (Cahiers du cinéma, $\mathrm{n}^{\circ} 120,1961$, p. 54).

4 On se souvient des incursions rapides et incisives de Pascal Bonitzer, Serge Daney, Jean-Pierre Oudart, Jean-Louis Comolli sur des questions de théorie comme le rapport son/image, la suture, le gros plan, le décadrage, le plan-tableau, le rapport fiction / documentaire, etc.

5 Ni André Bazin ni Serge Daney, même si tous les deux en ont eu le projet à la fin de leur vie, n'ont eu le temps d'écrire "le" livre de leur pensée du cinéma.

\section{OUVRAGES CITÉS}

Barthes, Roland. Roland Barthes par Roland Barthes. Paris: Seuil, 1975.

Daney, Serge. "Lire notre critique ci-dessous ". Libération (31 mars 1992).

Daney, Serge. "Le point critique». Cahiers du cinéma, n³ 356 (1984). 